\title{
Variables asociadas con el fenómeno de la deserción de los estudiantes en la Fundación Universitaria Konrad Lorenz
}

\author{
Johanna Barrero*, Gustavo Garzón ${ }^{\star *}$, Óscar Gómez ${ }^{\star \star *}$
}

* Magíster en Psicología Clínica. Consultora en procesos de mejoramiento y apoyo en el Área de Talento Humano. Jefe de la Oficina de Información y Evaluación, Fundación Universitaria Konrad Lorenz.

Correo electrónico: johanna.barrero@konradlorenz. edu.co

** Especialista en Sistemas Gerenciales de Ingeniería.

Correo electrónico: gustavoa.garzono@konradlorenz. edu.co

*** Magíster en Ciencias de la Educación con Mención en Docencia e Investigación Universitaria, Universidad Central de Chile. Director del Centro de Consejería Académica, Fundación Universitaria Konrad Lorenz. Correo electrónico: oscari.gomezr@konradlorenz.edu.co

Recibido: 9 de julio del 2013 Aceptado: 30 de octubre del 2013

Cómo citar este artículo: Barrero, J., Garzón, G. y Gómez, Ó. (2013). Variables asociadas con el fenómeno de la deserción de los estudiantes en la Fundación Universitaria Konrad Lorenz. Pensando Psicología, 9(16), 55-68.

\section{Resumen}

Los avances investigativos sobre deserción reflejan múltiples variables causales de dicho fenómeno, variables sociales, económicas y académicas, entre otras. No existen perfiles definidos para describir y explicar con precisión la forma en que se comportan cada una de estas variables por la heterogeneidad de población. La presente investigación, cuyo objetivo general fue identificar las variables sociodemográficas, financieras y académicas relacionadas con la deserción de los estudiantes en la Fundación Universitaria Konrad Lorenz, es un estudio ex post facto retrospectivo de grupo único, cuyo diseño estuvo orientado a reconstruir los hechos relacionados con la deserción estudiantil de la institución en las cohortes 2005-II, 2006-I, 2006-II y 2007-I, estableciendo posibles relaciones con variables sociodemográficas, financieras y académicas. La población objeto de estudio la constituyeron los estudiantes de pregrado de las cohortes mencionadas de cuatro programas de pregrado, sumando un total de 846 estudiantes. Los resultados indican que la deserción estudiantil en la institución ocurre por razones académicas, específicamente por el promedio acumulado que mostró poder predictivo para los estudiantes que desertan, y no necesariamente por otras variables de tipo socioeconómico y sociodemográfico, siendo los hombres quienes porcentualmente desertan más, aunque son más las mujeres las que ingresan a los programas profesionales ofertados.

Palabras clave: deserción, deserción en educación superior, estudios correlacionales sobre deserción, variables sociodemográficas, académicas y socioeconómicas.

\section{Variables Associated with the Phenomenon of Desertion of Students at the Fundación} Universitaria Konrad Lorenz

Abstract

Advances in research into university desertion reveal multiple social, economic and academic causes, among others, of this phenomenon. However, the university population is heterogeneous and there are no defined profiles that can describe and accurately explain how each factor behaves. The Spadies system at the Colombian Ministry of National Education aims to analyze desertion rigorously; however, universities have to generate their own study processes. It is within this context that the Konrad Lorenz Foundation University is conducting this research, whose general objective is to identify the socio-demographic, financial and academic variables associated with student desertion at this higher education institution. This is an ex- post- facto retrospective single-group study. Its design is focused on reconstructing the events associated with student desertion at this institution among semester II of 2005, I and II of 2006 and I of 2007-II cohorts, by establishing possible relationships to sociodemographic, financial and academic variables. The population under study consists of a total of 846 undergraduate students from the abovementioned cohorts of the Systems Engineering, Mathematics, International Business Administration and Psychology programs. The results indicate that student desertion at this institution stems from academic reasons, particularly those associated with the cumulative grade average. This points to a predictive power for students who drop out, but not necessarily in relation to other socio-economic and socio- demographic variables. According to this study, the percentage of men who drop out is higher than that of women, even though more women enroll in the professional programs offered.

Keywords: desertion, desertion in higher education, correlational studies on desertion, sociodemographic, academic and socioeconomic variables.

Variáveis associadas ao fenômeno da deserção dos estudantes na Fundação Universitária Konrad Lorenz

Resumo

Os avanços investigativos sobre deserção refletem múltiplas variáveis causais desse fenômeno, variáveis sociais, econômicas e acadêmicas, entre outras. Não existem perfis definidos para descrever e explicar com precisão a forma na qual se comportam cada uma dessas variáveis pela heterogeneidade de população. A presente pesquisa, cujo objetivo geral foi identificar as variáveis sociodemográficas, financeiras e acadêmicas relacionadas com a deserção dos estudantes na Konrad Lorenz, é um estudo ex post facto retrospectivo de grupo único, cujo desenho esteve orientado a reconstruir os fatos relacionados com a deserção estudantil da instituição nas coortes 2005-II, 2006-I, 2006-II e 2007-I, estabelecendo possíveis relações com variáveis sociodemográficas, financeiras e acadêmicas. O público-alvo deste estudo foi constituído por estudantes de graduação das coortes mencionadas de quatro programas de graduação, que somou um total de 846 estudantes. Os resultados indicam que a deserção estudantil na instituição ocorre por razões acadêmicas, especificamente pela média acumulada que mostrou poder preditivo para os estudantes que desertam, e não necessariamente por outras variáveis de tipo socioeconômico e sociodemográfico, sendo os homens os que percentualmente desertam mais, embora sejam mais as mulheres as que ingressam nos programas profissionais ofertados.

Palavras-chave: deserção, deserção em educação superior, estudos correlacionais sobre deserção, variáveis sociodemográficas, acadêmicas e socioeconômicas. 


\section{Introducción}

Actualmente el tema de la educación concentra buena parte de las políticas de desarrollo social, científico, económico y cultural de los países de todo el mundo, políticas internacionales derivadas de organismos como el Banco Mundial y Naciones Unidas (oNU - Unicef) entre otros, que promueven la puesta en marcha de investigaciones, programas y experiencias que impacten de manera favorable el acceso de las personas de distintos sectores de la sociedad en el sistema educativo, $\mathrm{y}$ al mismo tiempo, que fomenten la permanencia o disminución de la deserción estudiantil, hecho particular que impacta justamente de manera negativa la economía y el desarrollo social si no se le interviene oportunamente.

Para el caso de Colombia, según datos del Ministerio de Educación Nacional, la cobertura en la Educación Superior para el 2011 fue de $40,3 \%$, equivalente a 1.820 .000 jóvenes que están estudiando en las IEs. En comparación con el 2010, se incrementó en un 3,2\% y el 59\% de la población aún se encuentra por fuera del sistema, equivalentes a 2.583.129 jóvenes entre los 17 y 21 años. En el 2011 la deserción anual bajó del 12,9\% al 11,8\%. La meta es llegar al 9\% en el 2014 (Campo, 2012). En tal sentido, para la Fundación Universitaria Konrad Lorenz la investigación educativa en torno al fenómeno de la deserción y la permanencia constituye una base necesaria para el diseño y la ejecución de políticas pertinentes y los resultados.

De acuerdo con lo expuesto hasta aquí, particularmente con los datos específicos sobre deserción en Colombia y en la institución, se analiza la existencia de enfoques para el análisis de la deserción y retención que pueden ser agrupados en cinco categorías dependiendo del énfasis que otorgan a las variables explicativas, ya sea individuales, institucionales o del medio familiar. Las categorías para los enfoques son: psicológicas, económicas, sociológicas, organizacionales e interaccionistas (Braxton y cols., 1997, citados por Himmel, 2000).

Respecto de la generalidad de los enfoques existentes en la literatura sobre deserción, es posible señalar que la característica fundamental de los enfoques psicológicos se refiere a los rasgos de la personalidad que diferencian o discriminan a los alumnos desertores de los no desertores (Fishbein y Ajzen, 1975, citados por Torres, 2012). Este tipo de enfoques surgen de los modelos sociológicos que hacen énfasis en la influencia de los factores externos al individuo en la retención, adicionales a los psicológicos. El modelo de Spady (1970, citado por Torres, 2012) sugiere que la deserción es el resultado de la fal- ta de integración de los estudiantes con el entorno de la educación superior, y que el medio familiar es una de las muchas fuentes que expone al estudiante a influencias, expectativas y demandas, las que a su vez afectan el nivel de integración social en la universidad (Himmel, 2000).

Otra línea teórica acerca de los factores explicativos de la deserción es la línea económica que adopta el enfoque de costo-beneficio, postulando que cuando los beneficios sociales y económicos asociados a los estudios en la educación superior son percibidos como mayores que los derivados de actividades alternas, el estudiante opta por continuar en la universidad (Himmel, 2000).

El modelo de Tinto (2006-2007) que responde a la perspectiva organizacional, reconoce el rol de las instituciones en la generación de resultados. Este modelo postula que los estudiantes que presentan una integración social e intelectual con la institución, tienden a permanecer (Peterson 1993, citado por Donoso y Schiefelbein, 2007).

Finalmente, el modelo de Nora dentro del enfoque complementario o integrado, propuesto como referencia para el análisis de los resultados de la presente investigación en la Konrad Lorenz, relaciona las habilidades académicas de los estudiantes al ingresar en la universidad, las necesidades de apoyo financiero, los beneficios estudiantiles de la institución y el desempeño académico de los alumnos en ella, como factores determinantes de la deserción (Donoso y Schiefelbein, 2007). El modelo plantea que la persistencia en la educación superior se modela mediante un proceso que abarca tres etapas: una primera en la que la habilidad académica previa y los factores socioeconómicos afectan la disposición del estudiante para continuar con los estudios, así como las percepciones sobre las posibilidades de efectuarlos: la segunda etapa es aquella en la cual el estudiante estima los costos y beneficios asociados de sus estudios en una institución, lo que implica que al ingresar lo hace con un cierto compromiso inicial con la institución, el cual es revisado con el transcurrir del tiempo. En tercer lugar, derivada de las dos etapas anteriores, está la debida integración del estudiante al sistema educativo (Donoso y Schiefelbein, 2007).

De acuerdo con lo anterior, las variables estudiadas en la investigación fueron: a) variables predictoras: sociodemográficas (edad y sexo), variables financieras $\mathrm{y}$ variables académicas previas; $\mathrm{b}$ ) variable criterio: deserción (discriminada en deserción académica y deserción no académica).

La deserción, como variable criterio, se define como: "[...] un estudiante que ha abandonado durante dos o más periodos consecutivos la institución o no registra graduación $[\ldots]$... 
La deserción se discriminará en:

Deserción académica: hace referencia a los estudiantes que no continúan en la institución por haber perdido la calidad de estudiantes por bajo rendimiento académico. Según el Reglamento Académico de la Fundación Universitaria Konrad Lorenz (2012), las razones por las que un estudiante puede perder el cupo es por haber perdido cuatro o más asignaturas en un mismo periodo o por reprobar por tercera vez una asignatura. Deserción no académica: hace referencia a los estudiantes que no continúan en la institución durante dos o más periodos consecutivos, sin que hayan realizado procesos de aplazamiento, cancelación de semestre ni reingreso.

Entre las variables financieras se encuentran los créditos educativos. Dos de los principales determinantes del abandono de los estudios universitarios son el financiamiento del costo de la matrícula y los gastos de sostenimiento. Como medio de prevención de la deserción y como estrategia que permite la vinculación de personas de escasos recursos a la educación superior, surgen los créditos educativos, los cuales, en el caso colombiano, están liderados por el Instituto Colombiano de Crédito Educativo y Estudios Técnicos en el Exterior (Icetex), y cuyo impacto sobre la deserción es la disminución en un $25 \%$ de las probabilidades de deserción en los estudiantes (Ministerio de Educación Nacional, 2009).

Por otra parte, las ayudas proporcionadas a los estudiantes en forma de becas, se configuran como un factor de peso en las posibilidades de permanencia, en donde las tasas de deserción varían dependiendo de la cantidad y duración de la ayuda financiera con la que cuentan los estudiantes, e incluso en épocas de apuro económico el impacto sobre el abandono temprano de los estudios aumenta (Ishitani y DesJardins 2002; Ozga y Sukhmandan, 1998, citados por Díaz, 2008). Para la Konrad Lorenz, de acuerdo con lo estipulado conceptualmente en el Reglamento Académico (2005) sobre las becas, el Artículo 99 señala:

Se otorga al estudiante que cumple con: haber cursado al menos dos periodos académicos en la institución, haber tenido uno de los tres promedios más altos de la carrera, durante el periodo lectivo inmediatamente anterior; haber obtenido mención de honor en los dos periodos académicos inmediatamente anteriores; no tener sanciones disciplinarias durante su permanencia en la Facultad.

Para las variables académicas previas, en los primeros estudios realizados respecto a la deserción estu- diantil, se asumía que el principal determinante de la deserción eran aquellas variables relacionadas con aspectos netamente económicos; sin embargo, la evidencia empírica ha permitido, a través del seguimiento realizado a más de dos millones de estudiantes, postular que las condiciones académicas de entrada pueden ser más determinantes para pronosticar las probabilidades de deserción de los estudiantes universitarios (Ministerio de Educación Nacional, 2009).

Por esta razón, en la investigación se midió como variable histórica la calificación en las áreas del núcleo común de la prueba de Estado Saber 11 y el promedio académico acumulado a lo largo de su permanencia en la institución. Respecto de las competencias de la prueba Saber 11, se trata de un núcleo común integrado por las áreas de Lenguaje, Matemáticas, Biología, Química, Física, Filosofía, Ciencias Sociales e Inglés; asimismo existe un componente flexible en el que el estudiante elige entre seis (6) opciones disponibles a partir de sus intereses particulares. La prueba básicamente busca estudiar competencias referidas a los procesos que el evaluado debe realizar para dar solución a lo que plantea el cuestionario (Icfes, 2012).

Los componentes con que cuenta la prueba son ejes problemáticos, categorías de conceptos a partir de los cuales se formulan las preguntas. Estos ítems son diseñados de manera inherente a una disciplina o área del conocimiento particular.

\section{Objetivos}

\section{Objetivo general}

Determinar las variables sociodemográficas, financieras y académicas relacionadas con la deserción de los estudiantes en la Fundación Universitaria Konrad Lorenz.

\section{Objetivos específicos}

- Identificar las variables sociodemográficas asociadas con la deserción estudiantil en los estudiantes de pregrado de las cohortes 2005-II, 2006-I, 2006-II y 2007-I.

- Identificar las variables financieras asociadas con la deserción estudiantil en los estudiantes de pregrado de las cohortes 2005-II, 2006-I, 2006-II y 2007-I.

- Identificar las variables académicas asociadas con la deserción estudiantil en los estudiantes de pregrado de las cohortes 2005-II, 2006-I, 2006-II y 2007-I. 
- Establecer la relación de las variables sociodemográficas, financieras y académicas con la deserción de los estudiantes del programa de Psicología de la Fundación Universitaria Konrad Lorenz.

- Establecer la relación de las variables sociodemográficas, financieras y académicas con la deserción de los estudiantes del programa de Administración de Negocios Internacionales de la Fundación Universitaria Konrad Lorenz.

- Establecer la relación de las variables sociodemográficas, financieras y académicas con la deserción de los estudiantes del programa de Ingeniería de Sistemas de la Fundación Universitaria Konrad Lorenz.

- Establecer la relación de las variables sociodemográficas, financieras y académicas con la deserción de los estudiantes del programa de Matemáticas de la Fundación Universitaria Konrad Lorenz.

- Establecer el perfil de los estudiantes de pregrado que presentan deserción estudiantil en la Fundación Universitaria Konrad Lorenz.

\section{Método}

\section{Tipo de estudio}

Fue un estudio ex post facto retrospectivo de grupo único. El diseño estuvo orientado a reconstruir los hechos relacionados con la deserción estudiantil de la Fundación Universitaria Konrad Lorenz en las cohortes 2005-II, 2006-I, 2006-II y 2007-I, estableciendo posibles relaciones con variables sociodemográficas, financieras y académicas. En las dos primeras fases de la investigación se realizó el análisis descriptivo y correlacional de las variables predictoras y criterio en la población objeto de estudio; en la fase III se llevó a cabo un análisis de regresión logística multinomial para identificar la capacidad de predicción de las variables con la deserción, y en la fase IV se hizo la caracterización de los desertores académicos y no académicos de los programas de acuerdo con las variables predictoras.

\section{Población}

La población objeto de estudio fueron 846 estudiantes de las cohortes 2005-II, 2006-I, 2006-II y 2007-I de los programas de pregrado: Psicología, Administración de Negocios Internacionales, Ingeniería de Sistemas y Matemáticas de la Fundación Universitaria Konrad Lorenz.

\section{Procedimiento}

\section{Fase I}

Para la primera fase de la investigación se hizo la recolección de los datos por medio de los diferentes sistemas de información institucionales que son: Sistema Integrado de Información Institucional (sIII) específicamente los módulos de deserción y desempeño académico; Sistema de Registro Académico Escolaris, y Sistema Iceberg en lo relacionado con el módulo financiero. A nivel externo se usó el Spadies para revisar los datos concernientes al estrato y a la clasificación en el examen Saber 11.

\section{Fase II}

En la segunda fase de la investigación se llevó a cabo la sistematización de la información y los análisis descriptivos, correlacionales y predictivos entre las variables, por medio del programa estadístico spss.

\section{Fase III}

En la tercera fase se hizo la elaboración de la caracterización de los estudiantes que desertan y no desertan por programa, de acuerdo con las variables estudiadas.

\section{Resultados}

Los resultados se exponen a partir de dos tipos de análisis, inicialmente los análisis descriptivos de las variables por medio de medidas de tendencia central y de dispersión, según la escala de medida de las variables. Para los análisis correlacionales entre las variables sexo y financieras, los valores se obtienen a partir del coeficiente de correlación FI al nivel de significancia del 0,01. Para las variables edad, promedio y escalas del núcleo común del Saber 11 se adelantó el análisis por medio de la prueba $U$ de Mann-Whitney debido a la naturaleza continua de las variables predictoras y dicotomía de la variable criterio, junto con una distribución asimétrica entre las muestras, buscando identificar las diferencias entre las medias. Por último, se realizó una regresión logística multinomial para identificar la capacidad de predicción de las variables con la deserción. A continuación los resultados. 


\section{Resultados descriptivos relevantes}

En el figura 1 se presenta la distribución de la población objeto de estudio en las cohortes establecidas, observándose que el mayor número de estudiantes de ingreso, para todos los programas, se dio en el primer periodo del 2007 (252 estudiantes) y el menor número de estudiantes que ingresaron fue en el segundo periodo del 2005 (167 estudiantes).

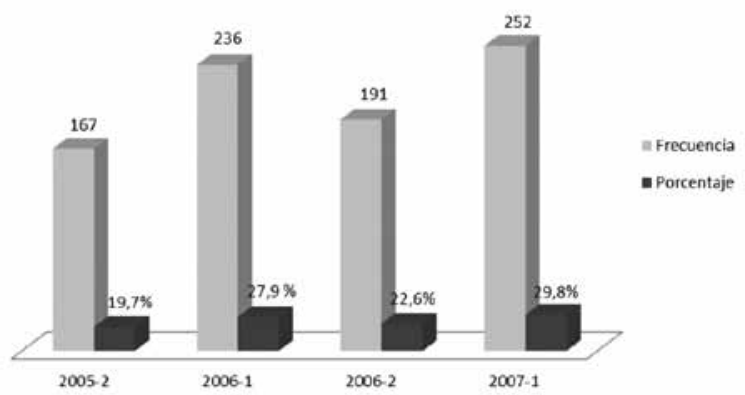

Figura 1. Estudiantes por cohorte y periodo académico Fuente: Base de datos institucional Escolaris 2012

Como se puede observar en la tabla 1, el 63,7\% de los estudiantes que ingresaron en las cohortes estudiadas son del programa de Psicología, y el 4,4\%, del programa de Matemáticas. Estos datos son coherentes con el tamaño de los programas a nivel institucional.

Tabla 1. Estudiantes por programa académico

\begin{tabular}{lcc}
\hline \multicolumn{1}{c}{ Programa académico } & Frecuencia & Porcentaje (\%) \\
\hline Ingeniería de Sistemas & 47 & 5,6 \\
\hline Matemáticas & 37 & 4,4 \\
\hline $\begin{array}{l}\text { Administración de Negocios } \\
\text { Internacionales }\end{array}$ & 223 & 26,4 \\
\hline Psicología & 539 & 63,7 \\
\hline Total & 846 & 100,0 \\
\hline
\end{tabular}

Fuente: Base de datos institucional Escolaris 2012

El figura 2 muestra que en la población estudiada predominan las mujeres, con un $64 \%$.

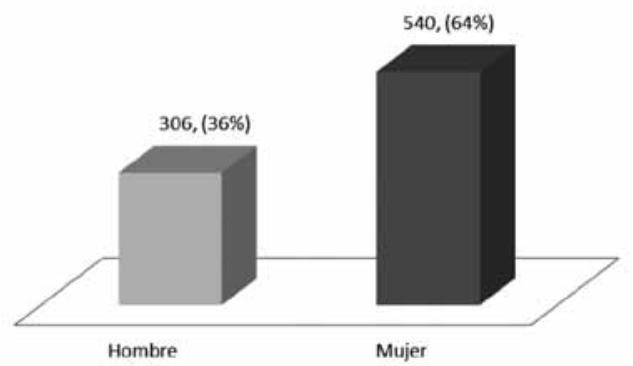

Figura 2. Distribución de la población por sexo Fuente: Base de datos institucional Escolaris 2012
De acuerdo con la tabla 2, el 83,2\% de los estudiantes ingresó en la institución con una edad que oscila entre los 15 y los 25 años; el 16,8\% restante ingresó en edades superiores a los 26 años.

Tabla 2. Distribución de la población por edad

\begin{tabular}{rrrr}
\hline & Frecuencia & Porcentaje (\%) \\
\hline Válidos & 15 a 20 & 507 & 59,9 \\
\hline 21 a 25 & 197 & 23,3 \\
\hline 26 a 30 & 81 & 9,6 \\
\hline 31 a 35 & 43 & 5,1 \\
\hline Igual o mayor a 36 & 18 & 2,1 \\
\hline Total & 846 & 100,0 \\
\hline
\end{tabular}

Fuente: Base de datos institucional Escolaris 2012

La medición del estado académico de los estudiantes de las cohortes 2005-II, 2006-I, 2006-II y 2007-I se hizo con corte al segundo periodo del 2012. En el figura 3 se observa que de la población total, el mayor porcentaje es de los estudiantes que desertaron $(48,11 \%)$; de estos, el $26 \%$ desertó por razones académicas y el $22,10 \%$ por razones no académicas. El $44,56 \%$ de los estudiantes que ingresan se gradúan.

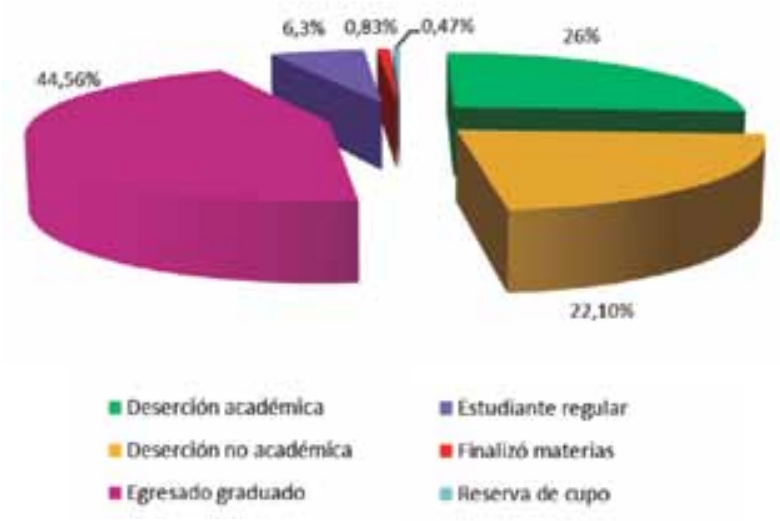

Figura 3. Distribución de la población por estado hasta el periodo 2012-2

Fuente: Base de datos institucional Escolaris 
En la tabla 3 se muestra la evolución de las cohortes objeto de estudio hasta el 2012-II. Se observa que la deserción académica es mayor a la deserción no académica en todos los programas, con excepción del de Matemáticas, que tiene una deserción no académica mayor. También se observa que la cantidad de estudiantes graduados es menor que la cantidad de desertores, con excepción del programa de Administración de Negocios Internacionales. En términos generales, el $48 \%$ de los estudiantes que ingresan desertan, y el $44,5 \%$ se gradúan.

Los datos muestran que el 0,5\% (4 estudiantes) de los estudiantes objeto de estudio obtuvo beca en algún momento de su proceso formativo. En la tabla 4 se observa que de estos cuatro (4) estudiantes, uno (1) desertó por razones académicas y tres (3) son egresados graduados.

En la tabla 5 se señala que el 58,6\% (496) de los estudiantes se sirvió de apoyos financieros en algún momento de su proceso formativo. De esta población que tomó apoyos financieros, el 24,19\% (120) desertó por razones académicas, el 21,57\% (107) lo hizo por razones no académicas, el 46,57\% (231) corresponde a egresados graduados, el 6,45\% (32) lo constituyen estudiantes regulares, el 1\% (5) finalizó materias pero no se graduó, y el $0,20 \%$ (1) restante tiene reserva de cupo a la fecha. A nivel descriptivo se observa que el 54,2\%
Tabla 3. Distribución de la población por estado y programa académico

\begin{tabular}{|c|c|c|c|}
\hline \multicolumn{3}{|c|}{ Programa académico } & $\begin{array}{l}\text { Periodo } \\
\text { académico }\end{array}$ \\
\hline \multirow{6}{*}{$\begin{array}{l}\text { Ingeniería de } \\
\text { Sistemas }\end{array}$} & \multirow{5}{*}{ Estado } & Deserción académica & 20 \\
\hline & & Deserción no académica & 14 \\
\hline & & Egresado graduado & 8 \\
\hline & & Estudiante regular & 3 \\
\hline & & Finalizó materias & 2 \\
\hline & \multicolumn{2}{|l|}{ Total } & 47 \\
\hline \multirow{5}{*}{ Matemáticas } & \multirow{4}{*}{ Estado } & Deserción académica & 13 \\
\hline & & \begin{tabular}{|l|} 
Deserción no académica \\
\end{tabular} & 17 \\
\hline & & \begin{tabular}{|l|} 
Egresado graduado \\
\end{tabular} & 6 \\
\hline & & Reserva de cupo & 1 \\
\hline & \multicolumn{2}{|l|}{ Total } & 37 \\
\hline \multirow{7}{*}{$\begin{array}{l}\text { Administración } \\
\text { de Negocios } \\
\text { Internacionales }\end{array}$} & \multirow{6}{*}{ Estado } & Deserción académica & 48 \\
\hline & & Deserción no académica & 42 \\
\hline & & \begin{tabular}{|l|} 
Egresado graduado \\
\end{tabular} & 121 \\
\hline & & Estudiante regular & 9 \\
\hline & & Finalizó materias & 2 \\
\hline & & Reserva de cupo & 1 \\
\hline & \multicolumn{2}{|l|}{ Total } & 223 \\
\hline \multirow{7}{*}{ Psicología } & \multirow{6}{*}{ Estado } & Deserción académica & 139 \\
\hline & & Deserción no académica & 114 \\
\hline & & \begin{tabular}{|l|} 
Egresado graduado \\
\end{tabular} & 242 \\
\hline & & \begin{tabular}{|l|} 
Estudiante regular \\
\end{tabular} & 39 \\
\hline & & Finalizó materias & 3 \\
\hline & & Reserva de cupo & 2 \\
\hline & \multicolumn{2}{|l|}{ Total } & 539 \\
\hline
\end{tabular}

Fuente: Base de datos institucional Escolaris 2012

Tabla 4. Distribución de la población por estado y recepción de becas a lo largo de su proceso formativo

\begin{tabular}{|c|c|c|c|c|c|}
\hline & & & \multicolumn{2}{|c|}{ Recibió o no becas } & \multirow{2}{*}{ Total } \\
\hline & & & No recibió & Recibió & \\
\hline \multirow{11}{*}{ Estado } & \multirow{2}{*}{ Deserción académica } & Recuento & 219 & 1 & 220 \\
\hline & & $\%$ dentro de estado & 99,5 & 0,5 & 100 \\
\hline & \multirow{2}{*}{ Deserción no académica } & Recuento & 187 & 0 & 187 \\
\hline & & $\%$ dentro de estado & 100 & 0,0 & 100 \\
\hline & \multirow{2}{*}{ Egresado graduado } & Recuento & 374 & 3 & 377 \\
\hline & & $\%$ dentro de estado & 99,2 & 0,8 & 100 \\
\hline & Estudiante regular & Recuento & 51 & 0 & 51 \\
\hline & \multirow{2}{*}{ Finalizó materias } & Recuento & 7 & 0 & 7 \\
\hline & & $\%$ dentro de estado & 100 & 0,0 & 100 \\
\hline & \multirow{2}{*}{ Reserva de cupo } & Recuento & 4 & 0 & 4 \\
\hline & & $\%$ dentro de estado & 100 & 0,0 & 100,0 \\
\hline \multirow{2}{*}{ Total } & & Recuento & 842 & 4 & 846 \\
\hline & & $\%$ dentro de estado & 99,5 & 0,5 & 100 \\
\hline
\end{tabular}

Fuente: Base de datos institucional Iceberg 2012

Tabla 5. Distribución de la población por estado y recepción de apoyos financieros a lo largo de su proceso formativo

\begin{tabular}{|c|c|c|c|c|c|c|}
\hline & & \multicolumn{4}{|c|}{ Tomó o no apoyos financieros } & \multirow{2}{*}{ Total } \\
\hline & & No tomó & $\%$ & Tomó & $\%$ & \\
\hline \multirow{6}{*}{ Estado } & Deserción académica & 100 & 28,57 & 120 & 24,19 & 220 \\
\hline & Deserción no académica & 80 & 22,86 & 107 & 21,57 & 187 \\
\hline & Egresado graduado & 146 & 41,71 & 231 & 46,57 & 377 \\
\hline & Estudiante regular & 19 & 5,43 & 32 & 6,45 & 51 \\
\hline & Finalizó materias & 2 & 0,57 & 5 & 1,01 & 7 \\
\hline & Reserva de cupo & 3 & 0,86 & 1 & 0,20 & 4 \\
\hline Total & & 350 & & 496 & & 846 \\
\hline
\end{tabular}

Fuente: Base de datos institucional Iceberg 2012 
de los estudiantes (269) no desertó y tomó apoyo financiero, y el 45,7\% (227 estudiantes) desertó habiendo tomado el mismo apoyo. Para el caso de quienes no tomaron apoyo financiero, 170 estudiantes permanecieron y 180 desertaron.

Como se observa en la tabla 6, los estudiantes del programa de Psicología son quienes tienen un mejor promedio acumulado a lo largo de la carrera $(3,4)$, seguido del programa de Administración de Negocios Internacionales $(3,38)$, en tercer lugar el programa de Matemáticas $(3,25)$ y por último el programa de Ingeniería de Sistemas $(2,9)$. Cabe anotar que existen promedios acumulados cercanos a cero (0), lo cual aumenta la desviación estándar.
Tabla 6. Promedio acumulado por programa

\begin{tabular}{lccc}
\hline \multicolumn{1}{c}{ Programa } & N & Media & Desv. típ. \\
\hline Ingeniería de Sistemas & 47 & 2,9189 & 1,04299 \\
\hline Matemáticas & 37 & 3,2562 & 0,98444 \\
\hline Negocios Internacionales & 223 & 3,3892 & 0,72157 \\
\hline Psicología & 539 & 3,4076 & 0,77088 \\
\hline
\end{tabular}

Fuente: Base de datos institucional Escolaris 2012

Haciendo el análisis del promedio de las áreas del núcleo común del Saber 11 por programa, en la tabla 7 se encuentra lo siguiente: para los programas de Ingeniería de Sistemas, Psicología y Administración de Negocios Internacionales, las áreas que tuvieron mayor puntaje neto fueron Lenguaje y Biología, y para el programa de Matemáticas los mejores puntajes

Tabla 7. Promedio acumulado y áreas del núcleo común del Saber 11 por programa

\begin{tabular}{|c|c|c|c|c|}
\hline & Programa & $\mathbf{N}$ & Media & Desv. típ. \\
\hline \multirow{8}{*}{ Ingeniería de Sistemas } & Promedio acumulado & 47 & 2,9189 & 1,04299 \\
\hline & Lenguaje & 47 & 52,28 & 10,812 \\
\hline & Matemáticas & 47 & 48,68 & 11,102 \\
\hline & Biología & 47 & 50,09 & 8,235 \\
\hline & Química & 47 & 48,72 & 12,356 \\
\hline & Física & 47 & 49,02 & 9,556 \\
\hline & Historia & 47 & 36,87 & 22,010 \\
\hline & Geografía & 47 & 29,36 & 24,183 \\
\hline \multirow{8}{*}{ Matemáticas } & Promedio acumulado & 37 & 3,2562 & 0,98444 \\
\hline & Lenguaje & 37 & 52,35 & 12,155 \\
\hline & Matemáticas & 37 & 51,86 & 10,771 \\
\hline & Biología & 37 & 54,08 & 8,532 \\
\hline & Física & 37 & 55,19 & 8,714 \\
\hline & Filosofía & 37 & 39,78 & 21,314 \\
\hline & Historia & 37 & 42,97 & 19,005 \\
\hline & Geografía & 37 & 37,46 & 22,431 \\
\hline \multirow{9}{*}{$\begin{array}{l}\text { Administración de Negocios } \\
\text { Internacionales }\end{array}$} & Promedio acumulado & 223 & 3,3892 & 0,72157 \\
\hline & Lenguaje & 223 & 52,47 & 7,856 \\
\hline & Matemáticas & 223 & 46,48 & 7,874 \\
\hline & Biología & 223 & 49,81 & 5,894 \\
\hline & Química & 223 & 46,14 & 6,761 \\
\hline & Física & 223 & 47,14 & 6,823 \\
\hline & Filosofía & 223 & 45,11 & 13,368 \\
\hline & Historia & 223 & 44,76 & 13,668 \\
\hline & Geografía & 223 & 37,21 & 20,147 \\
\hline \multirow{7}{*}{ Psicología } & Matemáticas & 539 & 45,26 & 7,857 \\
\hline & Biología & 539 & 49,71 & 7,016 \\
\hline & Química & 539 & 46,33 & 7,311 \\
\hline & Física & 539 & 47,04 & 7,534 \\
\hline & Filosofía & 539 & 41,79 & 17,600 \\
\hline & Historia & 539 & 44,05 & 13,818 \\
\hline & Geografía & 539 & 34,75 & 21,132 \\
\hline
\end{tabular}

Fuente: Sistema para el análisis de la deserción en Instituciones de Educación Superior sPADIEs 2012 
Tabla 8. Nivel de clasificación en la prueba Saber 11 por programa

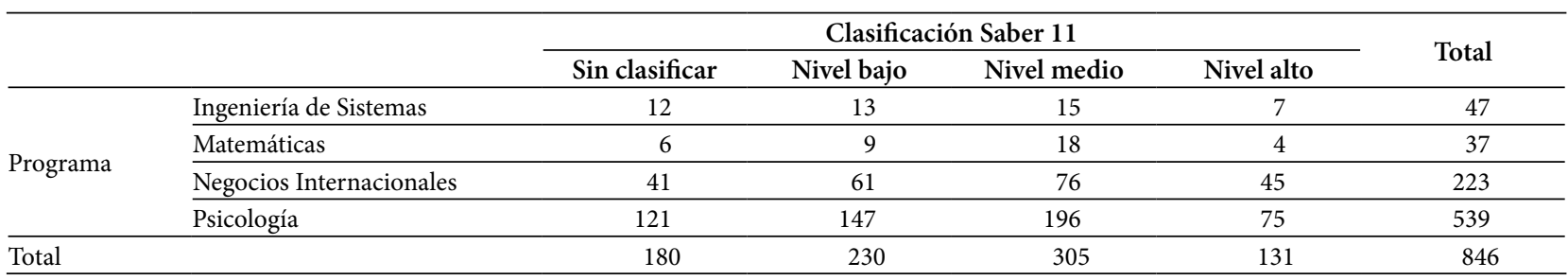

Fuente: Sistema para el análisis de la deserción en Instituciones de Educación Superior sPADIEs 2012

Tabla 9. Distribución de medias entre las variables cuantitativas de estudio

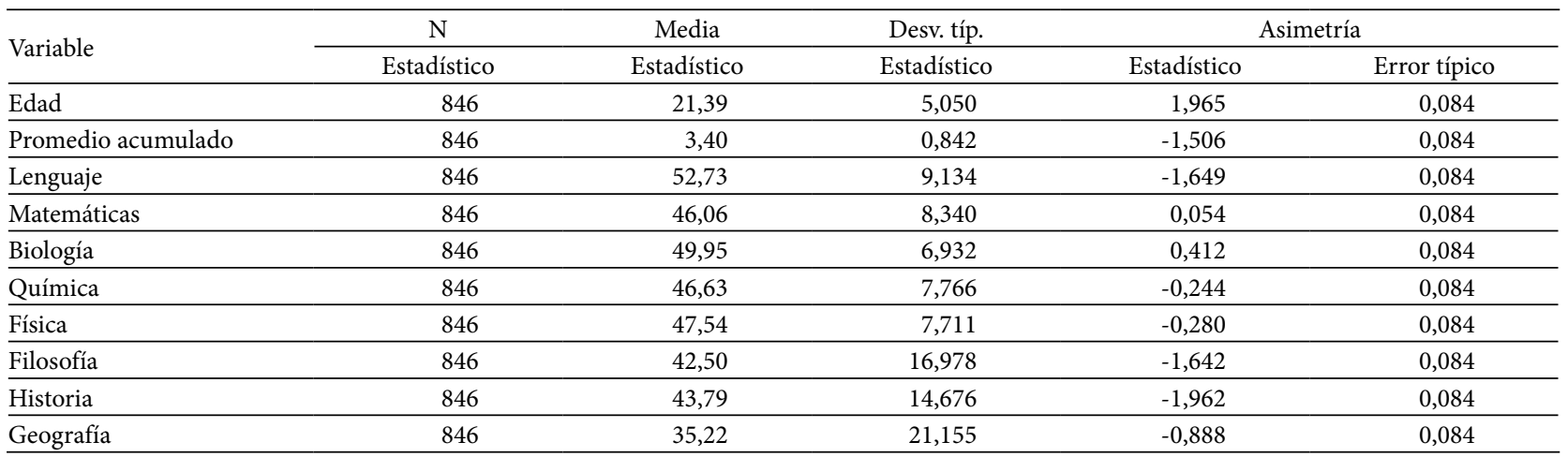

Fuente: Sistema para el análisis de la deserción en Instituciones de Educación Superior SPADIEs 2012

Tabla 10. Coeficiente de correlación FI entre sexo y variables financieras con la deserción por programa académico

\begin{tabular}{|c|c|c|c|c|}
\hline Programa académico & Variable & & Valor & Sig. Aproximada \\
\hline \multirow{3}{*}{ Ingeniería de Sistemas } & Sexo & Correlación FI & 0,059 & 0,685 \\
\hline & Recibió o no becas & Correlación FI & - & - \\
\hline & Tomó o no apoyos financieros & Correlación FI & 0,072 & 0,621 \\
\hline \multirow{3}{*}{ Matemáticas } & Sexo & Correlación FI & $-0,048$ & 0,771 \\
\hline & Recibió o no becas & Correlación FI & - & - \\
\hline & Tomó o no apoyos financieros & Correlación FI & $-0,414$ & 0,012 \\
\hline \multirow{3}{*}{$\begin{array}{l}\text { Administración de Negocios } \\
\text { Internacionales }\end{array}$} & Sexo & Correlación FI & $-0,208$ & 0,002 \\
\hline & Recibió o no becas & Correlación FI & $-0,042$ & 0,528 \\
\hline & Tomó o no apoyos financieros & Correlación FI & 0,043 & 0,517 \\
\hline \multirow{3}{*}{ Psicología } & Sexo & Correlación FI & $-0,087$ & 0,042 \\
\hline & Recibió o no becas & Correlación FI & - & - \\
\hline & Tomó o no apoyos financieros & Correlación FI & $-0,079$ & 0,065 \\
\hline
\end{tabular}

Fuente: resultados de la investigación "Variables asociadas con el fenómeno de la deserción de los estudiantes en la Fundación Universitaria Konrad Lorenz"

netos se obtuvieron en las áreas de Biología y Física. La desviación típica indica que hay mayor dispersión de los datos con relación a las medias para las áreas de Ciencias Sociales, y menor para las áreas de Ciencias Naturales; especialmente Biología es el área en la que menor desviación se presenta, y Geografía la de mayor dispersión. Se observa igualmente que el programa que tiene menos dispersión respecto de la media es Administración de Negocios Internacionales. 
La información que aparece en la tabla 8 indica que para todos los programas el mayor número de estudiantes tiene un nivel medio en la prueba Saber 11, y en segundo lugar están los niveles bajos en la prueba.

\section{Análisis correlacionales}

A continuación se presentan los resultados de los análisis correlaciones de las variables objeto de estudio. Para las variables sexo y financieras los valores se obtienen a partir del coeficiente de correlación FI al nivel de significancia del 0,01 .

Teniendo en cuenta que la variable criterio se expresa de manera dicotómica (desertó, no desertó) y las variables predictoras son de razón o intervalar, no existe un modelo correlacional que pueda establecer estadísticamente una relación. En razón a esto, para las variables edad, promedio y escalas del núcleo común de Saber 11 se adelantó el análisis por medio de la prueba U de Mann-Whitney debido a que la muestra no presenta una distribución normal, como se observa en la tabla 9, y para identificar las diferencias entre las medias.

Como se observa en la tabla 9, las variables no se distribuyen normalmente por estar por fuera del criterio de simetría $(-0,5$ a 0,5$)$ y ello corresponde con el supuesto requerido para adelantar el análisis de la prueba U de Mann-Whitney.

Como se observa en la tabla 10 , no existe correlación significativa entre la deserción y las variables predictoras sexo y apoyos financieros.

En la tabla 11 se presentan los resultados de la prueba U de Mann-Whitney, a partir de la cual se establecen las diferencias entre las medias de los puntajes de la edad, promedio acumulado y áreas del núcleo común de Saber 11 entre los estudiantes que desertan y no desertan.

En los análisis descriptivos se encuentran las medias con las cuales se hicieron las comparaciones con la prueba U de Mann-Whitney, encontrándose los siguientes resultados:

- Para el programa de Ingeniería de Sistemas existen diferencias significativas en la distribución de las medias para las variables promedio acumulado $(\mathrm{Pv}$ $=0,000)$ y el área de Historia $(\mathrm{Pv}=0,008)$ de Saber 11 con la deserción; es decir, los estudiantes que desertan tienen un promedio acumulado menor $(2,58)$ que los estudiantes que no desertan $(3,80)$, y quienes no desertan tienen una media mayor en el área de Historia $(51,00)$ de quienes desertan $(31,47)$.
Tabla 11. Prueba U de Mann-Whitney entre la edad y variables académicas con la deserción por programa académico

\begin{tabular}{|c|c|c|}
\hline $\begin{array}{l}\text { Programa } \\
\text { académico }\end{array}$ & Variable & Sig. \\
\hline \multirow{10}{*}{$\begin{array}{l}\text { Ingeniería } \\
\text { de Sistemas }\end{array}$} & Promedio & 0,000 \\
\hline & Edad & 0,064 \\
\hline & Lenguaje & 0,186 \\
\hline & Matemáticas & 0,685 \\
\hline & Biología & 0,164 \\
\hline & Química & 0,626 \\
\hline & Física & 0,943 \\
\hline & Filosofía & 0,756 \\
\hline & Historia & 0,008 \\
\hline & Geografía & 0,759 \\
\hline \multirow{10}{*}{ Matemáticas } & Promedio & 0,007 \\
\hline & Edad & 0,506 \\
\hline & Lenguaje & 0,801 \\
\hline & Matemáticas & 0,460 \\
\hline & Biología & 0,415 \\
\hline & Química & 0,560 \\
\hline & Física & 0,135 \\
\hline & Filosofía & 0,160 \\
\hline & Historia & 0,697 \\
\hline & Geografía & 0,469 \\
\hline \multirow{10}{*}{$\begin{array}{l}\text { Administración } \\
\text { de Negocios } \\
\text { Internacionales }\end{array}$} & Promedio & 0,000 \\
\hline & Edad & 0,002 \\
\hline & Lenguaje & 0,200 \\
\hline & Matemáticas & 0,789 \\
\hline & Biología & 0,056 \\
\hline & Química & 0,007 \\
\hline & Física & 0,016 \\
\hline & Filosofía & 0,043 \\
\hline & Historia & 0,119 \\
\hline & Geografía & 0,220 \\
\hline \multirow{10}{*}{ Psicología } & Promedio & 0,000 \\
\hline & Edad & 0,002 \\
\hline & Lenguaje & 0,002 \\
\hline & Matemáticas & 0,031 \\
\hline & Biología & 0,000 \\
\hline & Química & 0,011 \\
\hline & Física & 0,026 \\
\hline & Filosofía & 0,435 \\
\hline & Historia & 0,000 \\
\hline & Geografía & 0,002 \\
\hline
\end{tabular}

${ }^{\star *}$ El nivel de significancia es de 0,05

Fuente: resultados de la investigación "Variables asociadas al fenómeno de la deserción de los estudiantes en la Fundación Universitaria Konrad Lorenz"

- En el programa de Matemáticas se halló que la distribución del promedio no es la misma para los estudiantes que desertaron y no desertaron; es decir, existe significancia $(\mathrm{Pv}=0,007)$ y por tanto los estudiantes que desertan tienen un promedio acumulado menor $(3,08)$ que los que no desertan $(4,01)$.

- Para el programa de Administración de Negocios Internacionales existen diferencias significativas en la distribución de las medias para las variables promedio $(\mathrm{Pv}=0,000)$, edad $(\mathrm{Pv}=0,002)$ y las áreas de Química $(\mathrm{Pv}=0,007)$, Física $(\mathrm{Pv}=0,016)$ y Filosofía 
$(\mathrm{Pv}=0,043)$ de Saber 11 con la deserción. Los estudiantes que desertan tienen un promedio menor $(2,79)$ que los estudiantes que no desertan $(3,78)$ y una edad mayor (21 años) que los que no desertan (19 años). Asimismo, los estudiantes que desertan tienen una media menor en Física $(45,76)$, Química $(44,61)$ y Filosofía $(42,49)$ que los estudiantes que no desertan; Física $(48,08)$, Química $(47,17)$ y Filosofía $(46,88)$.

- Finalmente, para el programa de Psicología el promedio y las áreas del núcleo común de Saber 11, a excepción de Filosofía, evidencian diferencias significativas entre los estudiantes que desertan y no desertan. Los estudiantes que desertan tienen una media menor en promedio $(2,90)$ que los que no desertan $(3,84)$. Para las áreas del núcleo común de Saber 11, los estudiantes que desertan tienen una media menor en Lenguaje $(51,75)$, Matemáticas $(44,55)$, Biología $(48,66)$, Química $(45,57)$, Física
$(46,29)$, Historia $(42,04)$ y Geografía $(32,40)$ que los estudiantes que no desertan; Lenguaje $(53,92)$, Matemáticas (45,89), Biología (50,63), Química $(47,01)$, Física $(47,71)$, Historia $(45,82)$ y Geografía $(36,84)$. La edad mostró diferencias entre los desertores y no desertores, siendo los desertores quienes tienen menor edad que quienes se quedan.

\section{Análisis predictivos}

Para el análisis predictivo de las variables sociodemográficas, financieras y académicas con la deserción, se corrió una regresión logística multinomial es:

El modelo logístico resultante es:

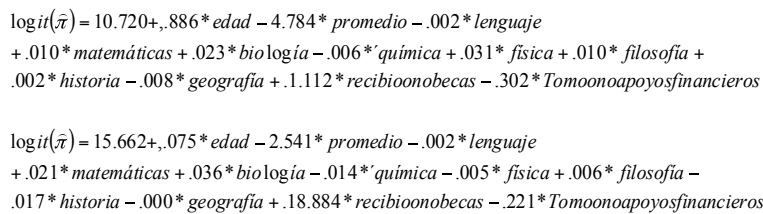

Tabla 12. Regresión logística multinomial entre las variables sociodemográficas, financieras y académicas con la deserción

\begin{tabular}{|c|c|c|c|c|c|c|c|c|c|}
\hline \multirow{2}{*}{ Estado } & \multirow{2}{*}{ Variables predictoras } & \multirow{2}{*}{ B } & \multirow{2}{*}{$\begin{array}{c}\text { Error } \\
\text { típ. }\end{array}$} & \multirow{2}{*}{ Wald } & \multirow{2}{*}{ gl } & \multirow{2}{*}{ Sig. } & \multirow{2}{*}{$\operatorname{Exp}(B)$} & \multicolumn{2}{|c|}{$\begin{array}{c}\text { Intervalo de confianza al } \\
95 \% \text { para } \operatorname{Exp}(B)\end{array}$} \\
\hline & & & & & & & & $\begin{array}{l}\text { Límite } \\
\text { inferior }\end{array}$ & $\begin{array}{l}\text { Límite } \\
\text { superior }\end{array}$ \\
\hline \multirow{13}{*}{ Deserción académica } & Intersección & 10,720 & 1,635 & 42,984 & 1 & 0,000 & & & \\
\hline & Edad & 0,086 & 0,034 & 6,165 & 1 & 0,013 & 1,089 & 1,018 & 1,165 \\
\hline & Promedio & $-4,784$ & 0,319 & 224,701 & 1 & 0,000 & 0,008 & 0,004 & 0,016 \\
\hline & Lenguaje & $-0,002$ & 0,016 & 0,014 & 1 & 0,905 & 0,998 & 0,967 & 1,030 \\
\hline & Matemáticas & 0,010 & 0,019 & 0,290 & 1 & 0,591 & 1,010 & 0,974 & 1,048 \\
\hline & Biología & 0,023 & 0,025 & 0,855 & 1 & 0,355 & 1,023 & 0,975 & 1,075 \\
\hline & Química & $-0,006$ & 0,023 & 0,065 & 1 & 0,799 & 0,994 & 0,950 & 1,040 \\
\hline & Física & 0,031 & 0,021 & 2,123 & 1 & 0,145 & 1,031 & 0,989 & 1,075 \\
\hline & Filosofía & 0,010 & 0,010 & 0,907 & 1 & 0,341 & 1,010 & 0,990 & 1,030 \\
\hline & Historia & 0,002 & 0,011 & 0,051 & 1 & 0,822 & 1,002 & 0,982 & 1,024 \\
\hline & Geografía & $-0,008$ & 0,007 & 1,112 & 1 & 0,292 & 0,992 & 0,978 & 1,007 \\
\hline & Recibió o no becas & 1,112 & 2,021 & 0,303 & 1 & 0,582 & 3,040 & 0,058 & 159,641 \\
\hline & Tomó o no apoyos financieros & $-0,302$ & 0,263 & 1,313 & 1 & 0,252 & 0,740 & 0,442 & 1,239 \\
\hline \multirow{13}{*}{ Deserción no académica } & Intersección & 5,662 & 1,280 & 19,553 & 1 & 0,000 & & & \\
\hline & Edad & 0,075 & 0,026 & 8,363 & 1 & 0,004 & 1,078 & 1,025 & 1,135 \\
\hline & Promedio & $-2,541$ & 0,225 & 126,978 & 1 & 0,000 & 0,079 & 0,051 & 0,123 \\
\hline & Lenguaje & $-0,002$ & 0,012 & 0,019 & 1 & 0,891 & 0,998 & 0,975 & 1,022 \\
\hline & Matemáticas & 0,021 & 0,015 & 1,956 & 1 & 0,162 & 1,021 & 0,992 & 1,052 \\
\hline & Biología & 0,036 & 0,019 & 3,604 & 1 & 0,058 & 1,037 & 0,999 & 1,076 \\
\hline & Química & $-0,014$ & 0,017 & 0,637 & 1 & 0,425 & 0,986 & 0,953 & 1,020 \\
\hline & Física & $-0,005$ & 0,016 & 0,095 & 1 & 0,757 & 0,995 & 0,965 & 1,026 \\
\hline & Filosofía & 0,006 & 0,008 & 0,521 & 1 & 0,471 & 1,006 & 0,990 & 1,022 \\
\hline & Historia & $-0,017$ & 0,008 & 4,963 & 1 & 0,026 & 0,983 & 0,968 & 0,998 \\
\hline & Geografía & 0,000 & 0,006 & 0,007 & 1 & 0,935 & 1,000 & 0,988 & 1,011 \\
\hline & Recibió o no becas & $-18,844$ & 0,000 & . & 1 & . & 6,551E-009 & $6,551 \mathrm{E}-009$ & 6,551E-009 \\
\hline & Tomó o no apoyos financieros & $-0,221$ & 0,212 & 1,092 & 1 & 0,296 & 0,801 & 0,529 & 1,214 \\
\hline
\end{tabular}


De acuerdo con lo presentado en la tabla 12, la variable que resulta ser significativa, según el estadístico de Wald, es el promedio, para el cual se rechaza la hipótesis nula $\mathrm{Ho}$; las otras variables no son significativas.

Tabla 13. Prueba de ajuste del modelo de regresión logística multinomial

\begin{tabular}{lcccc}
\hline Modelo & $\begin{array}{c}\text { Criterio de } \\
\text { ajuste del } \\
\text { modelo } \\
-2 \text { log } \\
\text { verosimilitud }\end{array}$ & Chi-cuadrado & Gl & Contrastes de la razón \\
de verosimilitud & & Sig. \\
\hline $\begin{array}{l}\text { Sólo la } \\
\text { intersección }\end{array}$ & 1733,138 & & & \\
\hline Final & 1087,611 & 645,527 & 24 &, 000 \\
\hline
\end{tabular}

Fuente: resultados de la investigación "Variables asociadas con el fenómeno de la deserción de los estudiantes en la Fundación Universitaria Konrad Lorenz"

Refiriéndose a la variable que evidencia significancia, el modelo de Negelkerke $(0,613)$ resulta tener un buen ajuste.

La variable que alcanza poder predictivo con la deserción es el promedio acumulado, y, por tanto, se entiende que aquellos estudiantes con promedios bajos, menores a 3,0, tienen mayor riesgo de deserción que quienes tienen promedios acumulados superiores. Lo anterior confirma las diferencias significativas halladas entre el promedio y la deserción para todos los programas en la prueba U de Mann-Whitney.

\section{Características comunes de los estudiantes de la Fundación Universitaria Konrad Lorenz}

Entre los programas académicos es predominante la población menor de 20 años; en cuanto al sexo, los programas de Administración de Negocios Internacionales y Psicología cuentan con mayor población de mujeres, mientras que en los programas de Ingeniería de Sistemas y de Matemáticas es mayor la población de hombres. En sus antecedentes académicos previos, según los resultados de las pruebas Saber 11, los estudiantes tienen mejor puntaje en las competencias interpretativas, argumentativas y propositivas propias del área de Lenguaje; se caracterizan por tomar apoyos económicos para la financiación de sus estudios universitarios. Asimismo, a nivel académico todos los programas, a excepción de Ingeniería de Sistemas, alcanzan el promedio necesario para la aprobación de las asignaturas.
Características comunes de los estudiantes que desertan por razones académicas por programa

Los estudiantes que desertan por razones académicas del programa de Administración de Negocios Internacionales tienen en común ser menores de 20 años, y mayoritariamente hombres. En sus antecedentes académicos, previos al ingreso en la Fundación Universitaria Konrad Lorenz, la competencia con menor puntuación es Geografía, en la que el evaluado evidencia conocimientos en la adecuada utilización de recursos geográficos al servicio de la sociedad, y sus competencias con mejor puntuación en las pruebas Saber 11 son aquellas que dan cuenta de su capacidad para analizar y distinguir premisas en el contenido de un texto escrito, hallando argumentos y posibles soluciones a problemas específicos. El promedio acumulado a lo largo de la carrera no es el suficiente para la aprobación de asignaturas $(3,0)$. Respecto de las características comunes a nivel financiero, el 56,3\% financia sus estudios universitarios por medio de algún tipo de crédito.

Los estudiantes que desertan académicamente del programa de Psicología se caracterizan por ser mujeres que ingresaron en la universidad con una edad menor a 20 años. La competencia con menor puntuación es Geografía, en la que el evaluado manifiesta conocimientos en la adecuada utilización de recursos geográficos al servicio de la sociedad y competencias con mejor puntaje en habilidades comunicativas básicas evidenciadas en el puntaje de Lenguaje en la prueba Saber 11. En referencia al promedio obtenido a lo largo de sus estudios, el puntaje es menor a lo esperado $(3,0)$. A nivel financiero, el 56,1\% tomó algún tipo de crédito.

Los estudiantes del programa de Ingeniería de Sistemas que desertan por razones académicas son en su mayoría hombres menores de 25 años de edad, y la mitad de ellos han recibido apoyo financiero. Respecto a los puntajes de Saber 11, los estudiantes que desertan por razones académicas muestran menores puntajes en Geografía, en la que el evaluado evidencia conocimientos en la adecuada utilización de recursos geográficos al servicio de la sociedad y mayores habilidades en establecer relaciones entre nociones y conceptos provenientes de contextos propios de la Física y de otras áreas del conocimiento, utilizando su capacidad crítica para valorar la calidad de una información o de un mensaje y para asumir una posición propia. En referencia al promedio, los estudiantes no superan la puntuación exigida para su aprobación $(3,0)$. 
Los estudiantes del programa de Matemáticas que desertan académicamente son especialmente hombres menores de 20 años, y el 61,5\% utiliza apoyos financieros. A nivel académico su promedio acumulado no les permite la aprobación de las asignaturas, y en referencia a su historia académica previa, medida por los puntajes en las áreas del núcleo común de Saber 11, muestran menor habilidad en Geografía, en la que el evaluado evidencia conocimientos en la adecuada utilización de recursos geográficos al servicio de la sociedad y mayor habilidad en establecer relaciones entre nociones y conceptos de la Física.

A nivel general, y teniendo en cuenta los hallazgos en la regresión logística multinomial, la característica común con poder de predicción entre los estudiantes que desertan académicamente es el promedio acumulado, el cual, como se mencionó, es menor a lo establecido en el Reglamento Académico para la aprobación de asignaturas $(3,0)$.

\section{Discusión}

En la Fundación Universitaria Konrad Lorenz se sabe que una cantidad considerable de estudiantes recibe apoyos financieros para la financiación de sus carreras por medio de diferentes líneas de créditos institucionales o por entidades externas. Para las cohortes estudiadas la cobertura fue del $58,63 \%$ de los estudiantes sin que se tenga registro de cuántos de ellos presentaban condición de vulnerabilidad socioeconómica mientras cursaban sus estudios; al adelantar el análisis estadístico se encontró que este dato de cobertura no correlaciona con la deserción (Cofer 2001, citado por Pinto, 2007). Los modelos teóricos para el análisis de la deserción señalan que el efecto positivo depende de aspectos puntuales como son: aumentos cuantiosos en el dinero destinado a suministrar este tipo de apoyo de modo que alcancen una relación porcentual adecuada entre los estudiantes con menores y mayores ingresos; los apoyos financieros tienen un impacto mayor si se estudia con rigurosidad la necesidad específica del estudiante que solicita la ayuda (Himmel, 2000).

Los hallazgos del presente estudio indican que tampoco existe asociación entre el sexo y la deserción en la Fundación Universitaria Konrad Lorenz, lo que lleva a considerar otras variables que deben ser objeto de análisis en investigaciones posteriores, y que a la luz de la evidencia empírica previa indican algún tipo de asociación; estas otras variables se relacionan con las dimensiones psicológica y sociocultural (Mateus, Herrera, Perilla, Parra, y Vera, 2011).

Para esta investigación, los datos relacionados con la edad en los programas de Administración de Negocios Internacionales y Psicología se orientan en la misma dirección con las conclusiones de diversos estudios llevados a cabo en Colombia y en Latinoamérica, que destacan una asociación entre las dos variables mencionadas (edad y deserción) siendo los estudiantes de menor edad quienes desertan (Pinto, 2007; Torres, 2012). De acuerdo con la hipótesis que se mencionó, a partir de las diferencias de medias, en cuanto a que los estudiantes del programa de Administración de Negocios Internacionales con mayor edad tienen mayor riesgo de desertar, es importante anotar que en los análisis descriptivos las edades de la población oscilan entre 15 y 45 años de edad, mostrando mayor deserción entre los 15 y los 25 años. En el caso del programa de Administración de Negocios Internacionales, el promedio de edad de quienes desertan es de 21 años, y se encuentra en el intervalo de los 15 a 25 años. Es decir, que sí podría afirmarse de manera general que los estudiantes de menor edad desertan. La asociación encontrada sólo en los programas de Administración de Negocios Internacionales y Psicología puede deberse a que es en estos en los que se concentra el $90 \%$ de la población objeto de estudio.

En el presente estudio se hallaron diferencias significativas entre quienes desertan y no desertan, con las variables promedio acumulado y áreas del núcleo común de Saber 11 (Ingeniería de Sistemas: Historia; Administración de Negocios Internacionales: Química, Física y Filosofía; Psicología: Lenguaje, Matemáticas, Biología, Química, Física, Historia y Geografía). Ahora bien, de estas variables la única con poder predictivo con la deserción es el promedio acumulado; es decir, los estudiantes que tienen menor promedio desertan, lo cual permite considerar que el desempeño académico bajo o menor a la calificación institucional requerida para la aprobación de las asignaturas afecta la disposición del estudiante para continuar con sus estudios universitarios (John y cols., 2000, citado por Torres, 2012). El promedio en general para las cohortes y para los desertores coincide con los datos ofrecidos por los estudios sobre deserción a nivel nacional e internacional, y con la caracterización ofrecida por el Ministerio de Educación Nacional.

Si bien la historia académica previa, medida en este caso por los puntajes en las áreas del núcleo común 
de Saber 11, no genera una predicción de si el estudiante deserta o no deserta, sí se encontraron áreas del núcleo común que evidencian diferencias significativas en las medias de los estudiantes que desertan (menores) con los que no desertan (mayores) por programa, lo que se tiene en cuenta como diferencia entre los dos grupos. Estos resultados pueden evaluarse dentro del programa para determinar si se definen como variables de ingreso teniendo en cuenta los puntajes mínimos y máximos en cada una de las áreas. Sin embargo, para poder tomar esta determinación, se debe realizar el análisis de estas variables con nueva población para poder hacer algún tipo de generalización.

Uno de los objetivos del presente estudio fue la elaboración del "perfil" de los desertores por programa; no obstante, al encontrar predicción de una sola variable (promedio acumulado) con la deserción, no fue posible cumplir con este objetivo. Igualmente, se quiso hacer una caracterización de los estudiantes que desertan por razones académicas y no académicas, pero no hay suficientes variables que diferencien a los dos grupos, salvo el promedio acumulado, a partir del cual son los estudiantes que desertan académicamente quienes cuentan con un promedio menor.

\section{Conclusiones}

- La mayoría de los estudiantes que ingresan en la Fundación Universitaria Konrad Lorenz son del programa de Psicología, con un $63,7 \%$ en las cohortes objeto de investigación.

- Los estudiantes que más desertan son los del programa de Matemáticas, con el 81\% de deserción, y el programa que porcentualmente tiene menos desertores es Administración de Negocios Internacionales, con un $40 \%$.

- La mayoría de las personas que ingresan en la Konrad Lorenz son mujeres, con el 64\%.

- La deserción por sexo indica que son los hombres quienes porcentualmente desertan más, con un $58 \%$ (178 hombres desertores) de 306 que ingresaron a estudiar en las cohortes objeto de estudio.

- Las mujeres tienen una deserción del 42\%, y 129 de 540 mujeres desertaron en las cohortes objeto de estudio.

- La medición del estado académico de los estudiantes de las cohortes 2005-II, 2006-I, 2006-II y 2007-I se hizo a corte del segundo periodo del 2012. De la población total el mayor porcentaje es de los estu- diantes que desertaron (48,11\%); de estos, el $26 \%$ desertó por razones académicas y el 22,10\% por razones no académicas. El 44,56\% de los estudiantes que ingresan se gradúa.

- Los estudiantes del programa de Psicología son quienes tienen un mejor promedio acumulado a lo largo de la carrera $(3,4)$, seguido del programa de Administración de Negocios Internacionales $(3,38)$; en tercer lugar está el programa de Matemáticas $(3,25)$, y por último el programa de Ingeniería de Sistemas $(2,9)$. Cabe anotar que existen promedios acumulados cercanos a cero (0), lo cual aumenta la desviación estándar.

- Haciendo el análisis del promedio de las áreas del núcleo común de Saber 11 por programa, se encuentra lo siguiente: para los programas de Ingeniería de Sistemas, Psicología y Administración de Negocios Internacionales, las áreas que tuvieron mayor puntaje neto fueron Lenguaje y Biología, y para el programa de Matemáticas los mejores puntajes netos se obtuvieron en las áreas de Biología y Física. La desviación típica indica que hay mayor dispersión de los datos con relación a las medias para las áreas de Ciencias Sociales, y menor para las áreas de Ciencias Naturales; especialmente Biología es el área en la que menor desviación se presenta, y Geografía la que presenta mayor dispersión. Se observa igualmente que el programa que tiene menos dispersión respecto de la media es Administración de Negocios Internacionales.

- Al hacer los análisis referentes a las áreas del núcleo común de Saber 11, se incluyó un nuevo aspecto de estudio dentro de esta variable que fue la "clasificación" de los estudiantes en esta prueba. La información relacionada con la clasificación en la prueba Saber 11 se extrajo del sistema Spadies. De la población objeto de estudio, el 21,3\% de los estudiantes no reporta una clasificación en la prueba. El 36,1\% de la población se ubica en una clasificación media, y el $15,5 \%$ en una clasificación alta. De ese $36,1 \%$ de estudiantes, el 51,5\% (157 estudiantes) permaneció, a diferencia del nivel bajo, en el que hubo mayor porcentaje en los estudiantes que desertaron, con un $53,9 \%$ (124).

- Para todos los programas, el mayor número de estudiantes tiene un nivel medio en la prueba Saber $11, y$ en segundo lugar están los niveles bajos en la prueba.

- No se estableció como variable objeto de estudio el último semestre cursado por los estudiantes de las 
cohortes; sin embrago, para el equipo de investigación fue relevante hacer la descripción de esta variable para saber en qué semestre se presenta mayor deserción. Para este análisis no se tuvieron en cuenta 378 registros que fueron los que finalizaron sus estudios; es decir, el estado de "egresado graduado". El 46,29\% de los estudiantes que desertaron cursó hasta primero o segundo semestre, siendo el segundo semestre con el mayor número de estudiantes que cursaron hasta este nivel.

- La deserción estudiantil en la Fundación Universitaria Konrad Lorenz ocurre por razones académicas, y no necesariamente por otras variables de tipo socioeconómico y sociodemográfico, siendo los hombres quienes porcentualmente desertan más, aunque son más las mujeres las que ingresan en los programas profesionales ofertados. Lo anterior ratifica que los apoyos actuales de la Fundación Universitaria Konrad Lorenz, ofrecidos por parte del Centro de Consejería Académica, son pertinentes dado que fortalecen las condiciones académicas de ingreso a partir de pruebas objetivas en habilidades cognoscitivas y hábitos de estudios y del desempeño en el transcurso de los procesos de formación por medio de programas de adaptación a la vida universitaria, habilidades académicas, tutorías académicas y consejería individual.

- Se acepta la hipótesis alterna para la existencia de una diferencia significativa entre las áreas del núcleo común de Saber 11 (Ingeniería de Sistemas: Historia; Administración de Negocios Internacionales: Química, Física y Filosofía; Psicología: Lenguaje, Matemáticas, Biología, Química, Física, Historia y Geografía) y la deserción; los estudiantes con menor media en las áreas mencionadas tienen mayor riesgo de desertar.

- Se acepta la hipótesis alterna para el promedio acumulado y la deserción; los estudiantes con menor promedio tienen mayor riesgo de deserción.

- Se acepta la hipótesis nula entre recibir apoyos financieros y la deserción.

- Se acepta la hipótesis nula entre el sexo y la deserción.

- Se acepta la hipótesis alterna para la edad y la deserción en los programas de Administración de $\mathrm{Ne}$ gocios Internacionales y Psicología. Los estudiantes de Administración de Negocios Internacionales con mayor edad tienen mayor riesgo de desertar y los estudiantes de Psicología con menor edad tienen mayor riesgo de desertar.

\section{Referencias}

Campo, M. (2012). Avances en la política para incentivar la permanencia en educación superior. Ministerio de Educación Nacional. Ministra de Educación Nacional. 4 de Octubre de 2012. Recuperado de http://www.mineducacion.gov.co/1621/propertyvalue-39230.html

Diaz, C. (2008). Modelo conceptual para la deserción estudiantil universitaria chilena. Estudios Pedagógicos, 34(2), 65-86. Recuperado de http://www.scielo.cl/pdf/ estped/v34n2/art04.pdf

Donoso, S. y Schiefelbein, E. (2007). Análisis de los modelos explicativos de retención de estudiantes en la universidad: una visión desde la desigualdad social. Estudios Pedagógicos, 33(1), 7-27.

Fundación Universitaria Konrad Lorenz. (2012). Reglamento académico.

Himmel, E. (2000). Modelos de análisis de la deserción estudiantil en la educación superior. Calidad de la educación. Recuperado de http://www.inacap.cl/tportal/portales/ tp4964b0e1bk102/uploadImg/File/EducacionSuperior/desercion/1_\%20DesercionE_Himmel.pdf

Icfes. (2012). Saber 11. ¿Qué se evalúa? Recuperado de http:// www.icfes.gov.co/examenes/saber-110/informaciongeneral/que-se-evalua.

Mateus, M., Herrera, C., Perilla, C., Parra, G. y Vera, A. (2011). Factores presentes en la deserción universitaria de la Facultad de Psicología de la Universidad de San Buenaventura, sede Bogotá, en el periodo comprendido entre 1998-2009. Psicología Avances de la Disciplina, 5(1), 121-133.

Ministerio de Educación Nacional. (2009). Deserción estudiantil: en la educación superior colombiana, metodología de seguimiento, diagnóstico y elementos para su prevención. Recuperado de http://www.mineducacion. gov.co/sistemasdeinformacion/1735/articles-254702_ libro_desercion.pdf.

Pinto, M. (2007). Cuestión de supervivencia. Graduación, deserción y rezago en la Universidad Nacional de Colombia. Bogotá: Editorial Universidad Nacional de Colombia.

Tinto, V. (2006-2007). Research and Practice of Student Retention: What next? Journal of College Student Retention, 8(1), 1-19. Recuperado de http://www.uaa. alaska.edu/governance/facultysenate/upload/JCSR_ Tinto_2006-07_Retention.pdf

Torres, L. (2012). Retención estudiantil en la educación superior: revisión de la literatura y elementos de un modelo para el contexto colombiano. Bogotá: Editorial Pontificia Universidad Javeriana. 